\title{
Repeated mixing and isolation: Measuring chronic, intermittent stress in Holstein calves ${ }^{1}$
}

\author{
C. S. Wilcox, ${ }^{*}$ M. M. Schutz, ${ }^{*}$ M. R. Rostagno,† D. C. Lay Jr.,† and S. D. Eichert ${ }^{2}$ \\ *Department of Animal Sciences, Purdue University, West Lafayette, IN 47907 \\ †Livestock Behavior Research Unit, USDA-ARS, West Lafayette, IN 47909
}

\begin{abstract}
Objectives of this study were to determine the physiological effects of psychological stress applied to dairy calves and to test if molasses consumption could be used to validate that a stressed condition was achieved. Twenty male calves (3 wk old) received jugular catheters and were randomly assigned to control (CTR; n $=4$ pens of 1 calf per pen) or social stress treatments (STR; $\mathrm{n}=4$ pens of 4 calves per pen). The STR treatment included 5 cycles of 24 -h isolation followed by regrouping with unfamiliar animals for $48 \mathrm{~h}$ (over 15 d). An ACTH challenge (0.1 IU $/ \mathrm{kg}$ of body weight) was used to determine adrenal fatigue. Peak and total cortisol concentrations were greater for STR calves until the ACTH challenge. After the ACTH challenge, CTR calf cortisol increased and STR calf cortisol continued to decrease, suggesting adrenal fatigue. The number of calves that became positive for fecal shedding of Salmonella after the acute stress of being moved and the number of calves that were positive after the move decreased with each move. Fifty-six percent of STR calves changed from negative to positive for shedding after the first move compared with $18.75 \%$ of STR calves remaining negative after the third move. Difference in fecal shedding of Enterobacteriaceae from samples taken before and after moving calves on d 6 was less than that on d 2, 3, and 5. Leukocyte counts were not different, but trends for day effects were detected for neutrophil and monocyte percentages. Molasses consumption was greater for STR calves on d 2 and 11, as was total consumption. Latency to lie after eating also increased as the study progressed; STR calves required more time to lie after eating on d 12 than on $\mathrm{d} 3$, and latency to lie was greater for STR than CTR on d 4, 8 , 12, and 14. The STR calves also stood more than the
\end{abstract}

Received April 19, 2013

Accepted July 20, 2013.

${ }^{1}$ Mention of trade names or commercial products in this paper is solely for the purpose of providing specific information and does not imply recommendation or endorsement by the US Department of Agriculture.

${ }^{2}$ Corresponding author: Susan.Eicher@ars.usda.gov
CTR calves in the 4-h afternoon period on $\mathrm{d} 4,5,7$, and 14. However, during the 4-h morning observations on d 14 (ACTH challenge), CTR calves stood more than STR calves. This model induced chronic stress, as characterized by adrenal fatigue, which was confirmed by molasses consumption and behavior changes. Therefore, molasses consumption could be used to confirm social stress in experimental models.

Key words: bacteria, cortisol, dairy calf, stress

\section{INTRODUCTION}

Dairy calf management, as recommended by the Babcock Institute of the University of Wisconsin (Wattiaux, 2000), includes a period of isolation followed by regrouping. To reduce pathogen load originating from the adult animals or from cross-contamination among calves (McGuirk, 2008), dairy farmers frequently remove neonates from their dams between 1 and $12 \mathrm{~h}$ postpartum and rear them in individual hutches or pens (USDA-APHIS, 2008). Following this period of isolation, heifers are typically weaned at 6 to $8 \mathrm{wk}$ of age and placed into groups. Male calves used for dairy beef or veal are now grouped earlier (4 to $6 \mathrm{wk}$ ), with emphasis on social needs of calves, and are periodically regrouped according to size.

After weaning, calf management then follows a routine of repeated regrouping to maximize group homogeneity (Garnsworthy, 2005). Each of these activities - weaning (Hickey et al., 2003), transportation (Fazio and Ferlazzo, 2003), isolation (Moberg, 2000), mixing, and change of housing (Veissier et al., 2001) - is known to elevate circulating cortisol concentrations, an indicator of acute stress.

Acute stress is the physiological response to physical or psychological threats to an animal's homeostasis. As the animal perceives a threat, corticotropin-releasing factor is produced in the hypothalamus, initiating a cascade of events along the hypothalamic-pituitaryadrenal (HPA) axis. Stimulation of corticotropin-releasing factor receptors located in the anterior pituitary gland produces a release of ACTH and glucocorticoids such as cortisol from the adrenal gland. As part of the 
allostatic control of stress, cortisol regulates the release of glucose from the liver and inhibits the production of ACTH in the pituitary, ultimately decreasing the production of cortisol (Douglas, 2005). Mild acute stimulation of the HPA axis causes a transient spike in metabolism and the temporary suspension of digestion, growth, and repair (Sapolsky, 2004). If an animal has sufficient reserves, it can recover from these physiological changes.

During acute stress, elevated circulating cortisol concentrations activate glucocorticoid receptors in the liver that increase the expression of glucose-6-phosphatase, which in turn increases the production and secretion of glucose. Acute stress also increases the secretion of adrenaline, which stimulates the production of insulin. During chronic stress, biological reserves become depleted, leading to adrenal fatigue and hypoglycemia.

Another physiological consequence of prolonged $\mathrm{ACTH}$ production and chronic elevation of plasma cortisol concentration is the disruption of immune system functions. Cytoplasmic glucocorticoid receptors are found in almost all mammalian cells. The percentages of neutrophils and eosinophils increase with acute stress. In contrast, chronic elevation of ACTH concentration reduces the production of neutrophils (Bilandzic et al., 2006). Chronic suppression of the immune system shifts the leukocyte differential profile, rendering even the humoral immune system inefficient (Moberg, 2000).

In addition to the benefits of elevating the humoral immune response at the expense of cell-mediated immunity during acute stress (Bilandzić et al., 2006), changes in behavior during acute stress are beneficial for the animal. Increased serum cortisol increases the production and secretion of noradrenaline, which heightens attention. However, repeated stimulation of the hypothalamus and activation of amygdala neuronal cells modifies the morphology of dendrites in these brain regions. This altered morphology changes the sensitivity of neurons and may cause anxiety, increased aggression, and other behavioral changes associated with chronic stress (Cook and Wellman, 2004).

One behavioral change noted in studies of chronic stress in mice and rats is a change in voluntary consumption of sucrose (Pothion et al., 2004). Change in sucrose consumption is typically measured as a percentage of total liquid intake in a 2-bottle preference test (pure water vs. $10 \%$ sucrose solution; Pothion et al., 2004). This change, relative to baseline or control animals, is accepted as an indicator of stress and is used in rodents to validate experimental stress protocols (Brenes-Sáenz et al., 2006). It is unknown if dairy calves will modify their consumption of high-sugar feedstuffs as a reaction to stress, as seen in mice and rats. However, a preliminary study conducted in our laboratory dem- onstrated that upon experimentally inducing greater glucose demand with phlorizin, calves increased their molasses consumption (Wilcox et al., 2008). The observation that endogenous glucose demand is reflected in a change in sucrose consumption behavior suggested that stress may also modify the sucrose consumption behavior of dairy calves.

Changes in behavior, shifts in immune system profile, and HPA axis resistance to ACTH challenge are indicative of chronic stress and are evident in animals that have been exposed to repeated, intermittent, mild stress. For example, weekly regrouping of calves during an 11-wk study resulted in a diminished reaction to novel acute stressors and attenuated cortisol response to ACTH challenges, compared with group-stable controls (Raussi et al., 2004). Social isolation and regrouping are mild psychological stressors that may occur frequently over the life of a calf under current management recommendations. The objectives of this study were to determine physiological effects of psychological stress of isolation and regrouping applied to dairy calves and to test if molasses consumption could be used to validate that a stressed condition was achieved.

\section{MATERIALS AND METHODS}

\section{Animals}

All animal-related protocols were approved by the Animal Care and Use Committee of Purdue University. Twenty 3 -wk-old male calves were fed $2 \mathrm{~L}$ of colostrum twice within $24 \mathrm{~h}$ of birth. Calves with 24-h plasma protein concentrations of $>5.5 \mathrm{mg} / \mathrm{dL}$ (measured by refractometer, Brix, Atago, Bellevue, WA) were transported from a commercial herd (Strauss Veal Feeds Inc., North Manchester, IN) for $2 \mathrm{~h}$ to the Farm Animal Behavior Laboratory of the Livestock Behavior Research Unit (US Department of Agriculture Agricultural Research Service, West Lafayette, IN). Upon arrival of calves, BW and rectal temperature were recorded. Calves were housed for $3 \mathrm{~d}$ in individual pens that converted to group pens by removal of partitions. Calves were then catheterized with jugular venous catheters (Cook Veterinary Products, Bloomington, IN), which were maintained with sterile heparinized saline (4 U of heparin/L of saline; Abbott Laboratories, Abbott Park, IL). After 3 d, calves were assigned randomly to pens of group or individually housed calves. Four pens of 1 control (CTR) calf each and 4 pens of 4 stressed (STR) calves each were used. Two extra calves were kept in individual pens as replacements to maintain group size if STR calves had to be taken off study. Calves were fed a continuation of the diet and standard operating procedures from the veal farms from which 
they came were continued. Calves were fed calf milk replacer in buckets $(20 \% \mathrm{CP}$ and $20 \%$ fat, nonmedicated; Strauss Veal Feeds Inc.) reconstituted at $12.5 \%$ solids and at $10 \%$ of BW twice daily (0700 and $1800 \mathrm{~h} ; 20 \%$ of BW daily) and adjusted over time per the producer's protocol, and calves were allowed access to fresh water in buckets at all times. Because these were veal calves, no dry feed (starter grain) was offered.

\section{Chronic, Intermittent, Mild Stress Model}

Calves were subjected to 5 cycles of isolation and regrouping (STR) or remained in individual calf pens (CTR). After completion of all 5 cycles, all calves were challenged with $0.1 \mathrm{IU}$ of $\mathrm{ACTH} / \mathrm{kg}$ of $\mathrm{BW}$ (Sigma Aldrich, St. Louis, MO).

After preconditioning to the Livestock Behavior Research Unit facility, 5 groups of 4 calves consisting of 1 CTR group and 4 stressed groups were placed in $7.31-\mathrm{m}^{2}$ group pens with straw bedding. These 5 pens were visually and physically isolated from one another by a solid plastic wall (Agri-Plastics Corp., Grassie, ON, Canada). The pen for the CTR calves was divided into 4 equal pens with a 3 -rail iron fence. This allowed for auditory, visual, and limited physical contact between calves. However, it also allowed monitoring of individual molasses consumption. After $48 \mathrm{~h}$, the 16 calves from the 4 stress-treated pens were moved (by calf cart; Wiggens, Gersham, OR) into isolation pens. After $24 \mathrm{~h}, \mathrm{ST}$ calves were moved out of isolation pens and into group pens consisting of 4 unfamiliar calves. This cycle was repeated 5 times, with new groups of unfamiliar calves formed each time.

Isolation pens, constructed of solid plastic walls that created visual and physical isolation, were $1.4 \mathrm{~m}^{2}$ and bedded with straw. Isolation pens were located in a room adjacent to the room with CTR calf and grouphousing pens within the same facility. Calves were placed in these pens such that members who formed the previous group were not near each other and members of the next group to be formed were not near each other. Between each move, both group and isolation pens were cleaned, wiped with disinfectant cleaning solution $(0.4 \%$ vol/vol Rocal D-plus, Pfizer Inc., New York, NY), and bedded with fresh straw to rid pens of odors from familiar calves. Control calves were moved in the calf cart on the same day for an equal time as STR calves and returned to their pen each time. Calves were given access to liquid molasses (Evolved Habitats, New Roads, LA) in standard-size feed buckets while in isolation pens. Control calves also were given access to molasses in their home pens at that time $(24 \mathrm{~h})$. All calves had a 12-h light and 12-h dark daily cycle throughout the study.

\section{Collection of Peripheral Blood}

Blood samples were collected by a single person trained to restrain and collect blood via jugular catheters into 2 - $\mathrm{mL}$ tubes containing $25 \% \mathrm{~K}_{2}$-EDTA and placed on ice. Catheters were flushed with $12 \mathrm{~mL}$ of saline followed by $3 \mathrm{~mL}$ of heparinized saline $(100 \mathrm{~g} /$ $\mathrm{dL})$. Blood samples were collected at 15 and $0 \mathrm{~min}$ before calves being moved (baseline). After each move, blood samples were taken from calves immediately and then $15,30,60,90,120$, and 300 min after calves had been moved.

\section{ACTH Challenge}

Blood samples were taken in a similar manner for the ACTH challenge: 2 baseline samples before injection of ACTH and then 15, 30, 60, 90, 120, and 300 min after ACTH challenge. Initial blood samples were collected $48 \mathrm{~h}$ after the last cycle in group pens. Two milliliters of blood was collected $15 \mathrm{~min}$ and just before injection of $\mathrm{ACTH}(0.1 \mathrm{IU} / \mathrm{kg}$ of $\mathrm{BW}$ in $3 \mathrm{~mL}$ of normal saline; Sigma-Aldrich), which was delivered through the jugular catheter and followed by $6 \mathrm{~mL}$ of normal saline. This dose has been used previously in our laboratory to create a significant and predictable cortisol response (Lay et al., 2008). Stress-treated calves were in group pens and CTR calves were in single calf pens throughout the ACTH challenge.

\section{Fecal Sample Collection and Bacterial Evaluation}

Fecal samples were collected before and after each move. Individual fecal samples were collected directly from the rectum using sterile swabs (Johnson and Johnson, Langhorne, PA). One-gram samples were processed for isolation of Salmonella enterica. An additional 1-g sample was processed for enumeration of total Enterobacteriaceae.

For Salmonella isolation, individual 1-g fecal samples were initially enriched in tetrathionate broth (1:10 dilution; $37^{\circ} \mathrm{C}$ for $24 \mathrm{~h}$; Neogen Corp., Lansing, MI), transferred $(0.1 \mathrm{~mL})$ to $10 \mathrm{~mL}$ of Rappaport-Vassiliadis broth (Neogen Corp.), incubated at $42^{\circ} \mathrm{C}$ for $24 \mathrm{~h}$, and streaked on 4 selective solid media xylose-lysinetergitol-4 (Neogen Corp.) agar plates and incubated at $37^{\circ} \mathrm{C}$ for $24 \mathrm{~h}$. Red to yellow colonies with black centers were picked from xylose-lysine-tergitol-4 agar plates, streaked on Rambach agar (DRG International Inc., Mountainside, NJ) and incubated at $37^{\circ} \mathrm{C}$ for $24 \mathrm{~h}$. Red colonies were considered positive for Salmonella.

To enumerate total Enterobacteriaceae, individual samples were serially diluted (10-fold) in buffered peptone water (Neogen Corp.). One milliliter of each 
dilution was plated in duplicate on a $20-\mathrm{cm}^{2}$ all-in-one plating system (Petrifilm Enterobacteriaceae Count Plates, 3M Microbiology, St. Paul, MN) and incubated at $37^{\circ} \mathrm{C}$ for $24 \mathrm{~h}$. The number of colony-forming units (cfu/g) for each count plate was recorded.

\section{Environmental Measures}

The ambient temperature and relative humidity were recorded every $30 \mathrm{~min}$ for the $14 \mathrm{~d}$ of the study with data loggers (Hobo, MicroDAQ, Warner, NH). Data loggers were placed about $1 \mathrm{~m}$ above the floor at each end of the room with isolation pens. Another set of data loggers was placed about $1.3 \mathrm{~m}$ above the floor at the end of the room that housed CTR and group calves.

\section{Behavioral Measures}

Behavior of calves in group pens was recorded using ceiling-mounted cameras (VW-BP330, Panasonic Secaucus, NJ) and a digital video recording system (Xenon; Interpacific, Northbrook, IL). Behaviors were recorded continuously during the 12 -h light period throughout the study. Animal posture of laying or standing and activities, including walking, running, eating, drinking, resting, being alert, socially interacting, and self-grooming, were measured using a 10-min instantaneous scan sample technique (behaviors that occurred at the end of each 10-min period over the 12-h light cycle for each of the days). Latency to lie down after eating in the morning and evening was determined each day using continuous behavior observations. The percentage of observations in which calves were exhibiting play behavior was calculated.

Molasses was weighed in buckets and buckets were placed on hooks in each isolation pen so that molasses was available as soon as the calf entered the pen. At the same time, molasses in buckets was placed in CTR pens in place of milk buckets. The next morning just before feeding, each bucket with remaining molasses was weighed again. Consumption of molasses was calculated as the difference of those 2 weights.

\section{Enumeration and Composition of Blood Leukocyte Population}

Jugular blood collected into the EDTA tubes just before the calf being moved each time was set on a tube rocker and allowed to come to room temperature. The 5-part blood cell differential counts consisting of hematocrit percentages, total white blood cell counts, and counts and relative percentages of neutrophils, lymphocytes, monocytes, eosinophils, and basophils were determined using an automated cell counter (Hemavet HV950FS, Drew Scientific, Dallas, TX).

\section{Cortisol Assay}

Total circulating plasma cortisol concentration in each of the blood samples was measured by RIA (Coata-Count RIA Cortisol kit, Diagnostic Products Corp., Los Angeles, CA). The protocol has been previously validated in this laboratory for measuring bovine plasma cortisol concentrations (Eicher et al., 2007). Briefly, $500 \mu \mathrm{L}$ of buffer, $10 \mu \mathrm{L}$ of the calibrators, CTR and STR samples in duplicate, and $1.0 \mathrm{~mL}$ of ${ }^{125} \mathrm{I}$ cortisol was added to 4 uncoated $12 \times 75 \mathrm{~mm}$ polypropylene tubes (for total counts and nonspecific binding in duplicate) and cortisol-specific antibody-coated tubes. After vortexing each tube and incubating for $45 \mathrm{~min}$ at $37^{\circ} \mathrm{C}$, tube contents were decanted thoroughly and counted for 1 min using a gamma counter (2470 Wizard, Perkin Elmer, Waltham, MA). Sample concentrations were calculated from the logit-log representation of the calibration curve and were presented as the average nonspecific binding-corrected counts per min. Interassay CV was $2.12 \%$, and intraassay CV was $\leq 1.7 \%$. Samples with $\mathrm{CV}>5 \%$ were rerun.

\section{Statistical Analysis}

Two calves were kept in reserve as replacement stressed animals in the event that they were needed. The replacements were used before $\mathrm{d} 5$ and maintained the integrity of the experimental design so that at all times group pens could be regrouped with a total of 4 calves each unfamiliar to the other. However, blood and behavior samples were not collected from these replacement calves, so data analysis reflects a sample size of 14 for the stressed group after d 5. Animal numbers were determined for each study using variation in previous work and $\alpha=0.05$ in SAS Power test. All statistical analyses were conducted using the MIXED procedure of SAS, version 9.1 (SAS Institute Inc., Cary, NC). Calves nested within treatment were considered to be random. Normality of the data was checked using the UNIVARIATE procedure and residuals were examined using PROC GLM. When necessary, data were transformed to achieve normality and the statistical ANOVA and $P$-values reported were calculated using transformed data. Graphs are presented using least squares means of untransformed data. Treatment, day, and move were considered fixed effects for variables, except for cortisol data, where time and the interactions treated $\times$ day and treated $\times$ move were considered fixed effects. The area under the curve, determined by the concentration of cortisol found in each of the 9 blood samples taken 
on each of the move days, was calculated using the SAS macro published by Yeh (2002). Cortisol data required an inverse square root transformation, whereas the leukocyte data were nontransformed and molasses consumption data required a square root transformation. A lambda of -1 was the optimum transformation for normalizing the behavior data as found by the BOXCOX procedure of SAS. Probability values reported reflect statistical analysis of transformed data, and the more conservative Tukey adjustment was used. Correlation analysis was performed for $3 \mathrm{~d}$ of molasses consumption and latency to lie and for $4 \mathrm{~d}$ of molasses consumption and peak cortisol.

\section{RESULTS AND DISCUSSION}

\section{Environment}

On d 3 of the study, ambient temperatures increased to $37^{\circ} \mathrm{C}$ with relative humidity varying between 80.5 and $94.8 \%$ (every 15 min samples, $24 \mathrm{~h} / \mathrm{d}$, similar to environmental humidity). Barn temperatures rose briefly to $36.2^{\circ} \mathrm{C}$ until an effective cooling system maintained the barn temperature at $29.5^{\circ} \mathrm{C}$ or below with a mean daily temperature $27.1^{\circ} \mathrm{C}$ or below. Calf rectal temperatures did not differ between treatments, but varied by day (data not shown). The shedding of Enterobacteriaceae, neutrophil percentages, and percentage of observations spent standing reflect that temperature increased in STR and CTR calves.

\section{Cortisol}

Area under the curve of cortisol concentrations over the 315-min experimental period and peak cortisol and baseline cortisol concentrations (Figure 1) had a treatment $\times$ day interaction $(P<0.01)$. Area under the curve and peak cortisol concentrations for STR calves on $\mathrm{d} 1$ to 5 were greater than those of CTR calves and lower than those of CTR calves after ACTH injections. Baseline concentrations also had a treatment $\times$ day effect. However, for baseline values, CTR calves had greater cortisol concentrations on $\mathrm{d} 2$ and 3 but lesser concentrations throughout the remainder of the study.

Decreased cortisol concentration in response to a repeated stress stimulus can indicate adrenal fatigue or habituation (Moberg, 2000). Social stress of calves by isolation and regrouping in this study not only increased peak and total cortisol secretions but baseline concentration as well. When ACTH was injected, STR
A)

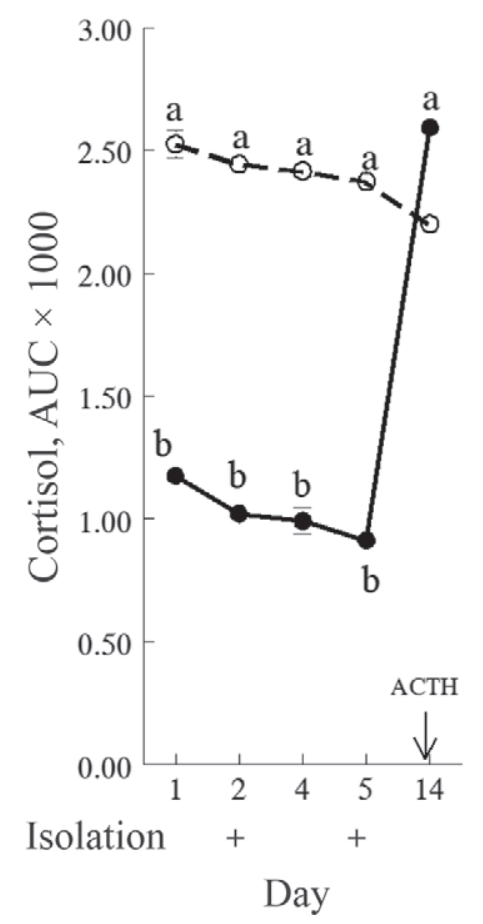

B)

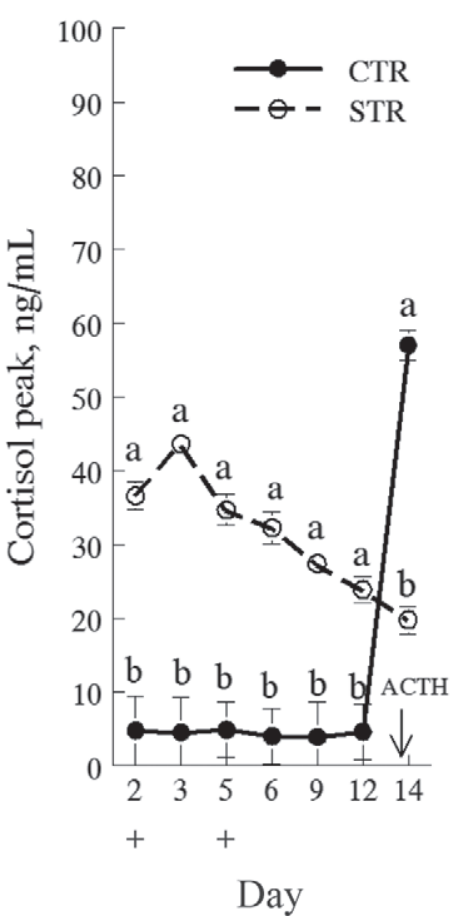

C)

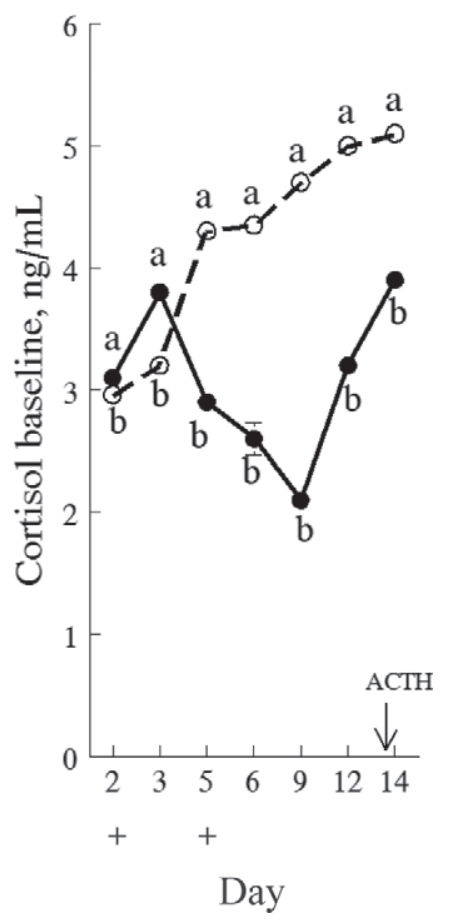

Figure 1. Mean area under the curve (AUC; A), peak (B), and baseline (C) cortisol concentrations by day for control (CTR) and social stress-treated calves (STR). The mean of the AUC derived from the cortisol concentration in blood samples taken after treatment, peak cortisol concentrations, and baseline cortisol concentrations had a treatment by day effect $(P=0.001)$. Graph means are nontransformed data. ${ }^{\mathrm{a}, \mathrm{b}} \mathrm{Means}$ with differing superscripts within a day are different $(P<0.05)$. Injections of ACTH are indicated by arrows. 
Table 1. Fecal shedding of Salmonella for stress-treated (STR; $\mathrm{n}=16)$ and control $(\mathrm{CTR} ; \mathrm{n}=4)$ calves before and after moving to new groups

\begin{tabular}{llccc}
\hline $\begin{array}{l}\text { Move } \\
\text { number }\end{array}$ & Treatment & $\begin{array}{c}\text { Shedding } \\
\text { pre-move }\end{array}$ & $\begin{array}{c}\text { Shedding } \\
\text { post-move }\end{array}$ & $\begin{array}{c}\text { Change: } \\
- \text { to }+(\%)\end{array}$ \\
\hline 1 & STR & 4 & 11 & $9(56)$ \\
& CTR & 3 & 3 & $1(25)$ \\
2 & STR & 0 & 3 & $3(19)$ \\
3 & CTR & 2 & 4 & $3(19)$ \\
4 & STR & 3 & 6 & $0(0)$ \\
4 & CTR & 1 & 1 & $1(6)$ \\
OTR & 0 & 1 & 0 \\
& CTR & 1 & $26^{\mathrm{A}}(0)$ \\
& STR & $7^{\mathrm{a}, \mathrm{A}}$ & $8^{\mathrm{a}, \mathrm{B}}$ & 3 \\
\hline
\end{tabular}

${ }^{\mathrm{a}, \mathrm{b}}$ Statistical comparison within the same row by $\chi^{2}(P<0.05)$.

${ }^{\mathrm{A}, \mathrm{B}}$ Statistical comparison between STR and CTR (within a column) by $\chi^{2}(P<0.05)$.

calves did not respond, but CTR calves responded with concentrations greater than or equal to those of STR calves during the stress events. These data show that adrenal fatigue was achieved using this model over 2 wk.

\section{Characterization of Blood Leukocyte Populations}

Hematocrit percentage (data not shown) tended to have $(P=0.08)$ a treatment $\times$ day effect. Hematocrit percentages were greater $(P=0.05)$ for CTR than for STR calves on d 9 (28.0 and $21.48 \%$, respectively). No treatment or treatment $x$ day effects were found for any of the leukocyte counts (data not shown). However, a day effect was found for percentages of neutrophils $(P=0.01)$, lymphocytes $(P=0.03)$, and monocytes $(P=0.02)$.

Because one focus of this study was on the effect of repeated isolation and mixing on the leukocyte profile, blood samples designated for this analysis were taken just before each move. The effect on the leukocyte profile of acute stress caused by the calf cart ride is, therefore, not evident from this data set. Increased cortisol concentrations are known to cause depressed lymphocyte counts (Riondato et al., 2008) and neutrophilia (Madsen-Bouterse et al., 2006). In this study, total leukocyte concentration did not change due to the calf being stressed. Because changes in neutrophil numbers are a later event than increases in cortisol concentrations after a stressor and can be observed at $24 \mathrm{~h}$ after the stressor, samples were collected only once on each day of blood collections.

\section{Salmonella and Enterobacteriaceae}

During the first move, 4 and 3 calves (STR and CTR, respectively) were positive for fecal shedding of Salmonella before the move and 11 and 3 were positive after the move. During the second move, 0 and 2 (STR and CTR, respectively) were positive for fecal shedding of Salmonella before the move, whereas 3 and 4 (STR and CTR, respectively) were positive after the move. Thereafter, the number of CTR calves that were positive after the move decreased with each move (Table 1 ). However, more STR calves (6) were positive after the third move and did not decrease until the fourth move. After the first move of group to isolation, $56.25 \%$ of STR calves and $50 \%$ of CTR calves changed from negative to positive for shedding. After the second move of isolation to group, $18.75 \%$ of stressed calves and $50 \%$ of CTR calves changed from negative to positive for shedding. After the third move from group to isolation, $18.75 \%$ of STR calves and no CTR calves changed from negative to positive for shedding of Salmonella.

A small number of calves (12.5\% for STR group and $25 \%$ for the CTR) changed from positive to negative on the first move (data not shown). Twenty-five percent of the CTR calves also changed from positive to negative for fecal shedding of Salmonella on the fourth move. Further analysis of Salmonella shedding was not done because the calves were treated for pneumonia.

The number of Enterobacteriaceae (cfu) found in fecal samples taken before each move (Figure 2A) showed treatment $(P=0.038)$ and day $(P=0.017)$ effects. The mean number of Enterobacteriaceae in the STR group before the move on $\mathrm{d} 3$ tended $(P=0.095)$ to be greater than that on $\mathrm{d} 2$, and was greater than on d $5(P=0.007)$ and d $6(P<0.001)$. Difference in the mean number of Enterobacteriaceae from fecal samples taken before and after moving calves, whether into or out of isolation pens (Figure 2A), followed the same pattern. Mean difference was significantly affected by day $(P=0.001)$, and a treatment $\times$ day interaction $(P$ $=0.023)$ was detected. On d 3 , difference in fecal shedding of Enterobacteriaceae for STR calves was greater $(P<0.001)$ than for CTR calves. 
A)

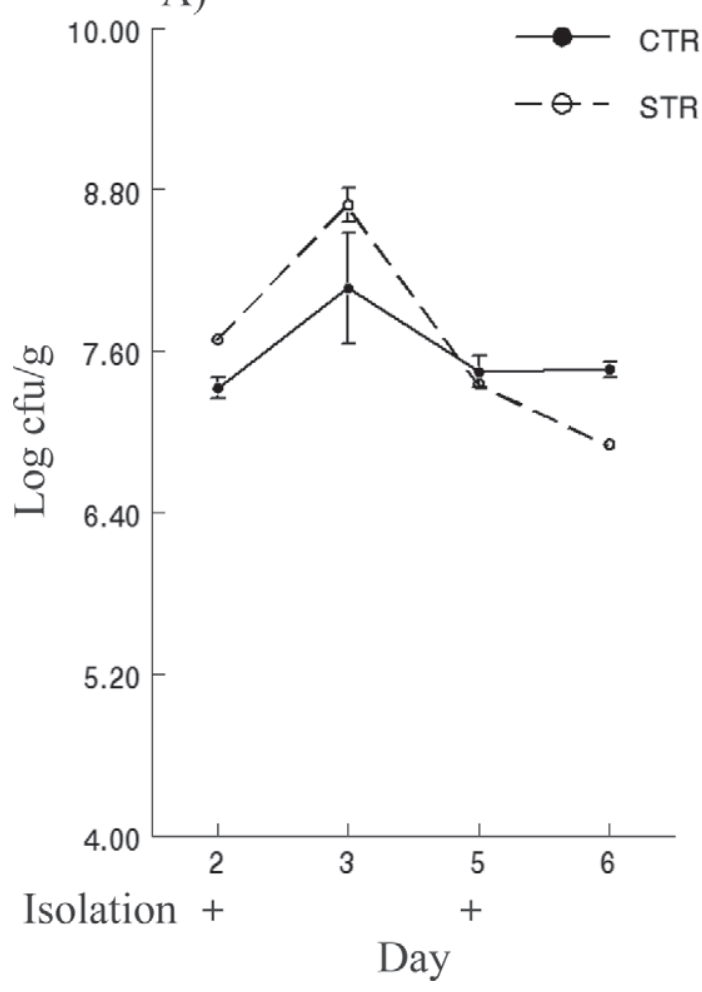

B)

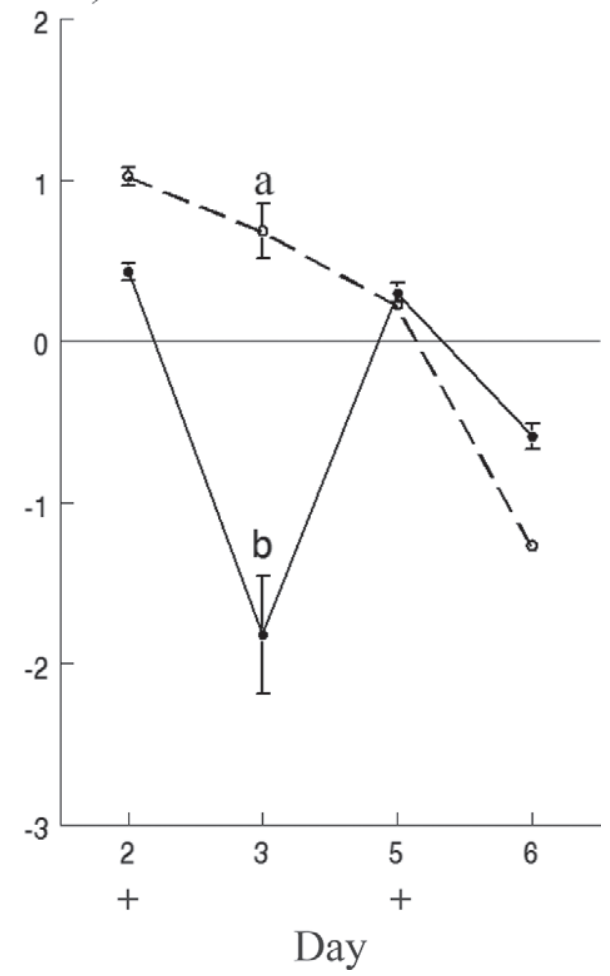

Figure 2. The mean number (cfu) of Enterobacteriaceae in fecal samples taken before each move (A) and the mean difference in number of Enterobacteriaceae in fecal samples taken before and after moving calves $(\mathrm{B})$. Graph means are nontransformed data. CTR $=$ control, $\mathrm{STR}=$ social stress treated. ${ }^{\mathrm{a}, \mathrm{b}}$ Means within a day with different letters differ $(P<0.05)$.

Habituation to acute stress was also evident in the pattern of fecal shedding of total Enterobacteriaceae, and of Salmonella in particular. Composition and quantity of pathogenic intestinal bacteria is known to increase because of stress (Kanitz et al., 2004; Williams et al., 2008). Fecal shedding of Salmonella is directly related to ileal loop concentration (Callaway et al., 2005). Both STR and CTR calves entered the study shedding approximately $107.5 \mathrm{cfu}$ of Enterobacteriaceae per gram of fecal matter. This high number might result from the stress of travel, as evidenced by 2 calves testing positive for Salmonella at that time, too. By the second move, the number of Enterobacteriaceae found in the fecal samples peaked and then steadily decreased over the next 2 moves, as did the difference between the number of Enterobacteriaceae before and after each move. Fecal shedding of Salmonella also followed this pattern. After the first move, 9 of 16 STR calves that were negative before the move tested positive for Salmonella in samples taken after the move. This declined rapidly over the next 3 moves until 1 out of the 16 STR calves changed from testing negative for fecal shedding of Salmonella before the move to being positive after the move. This pattern of declining differences of fecal shedding pre- and post-move confirmed the assumption that declining plasma cortisol concentration was due, at least in part, to habituation to the acute stressors. Fecal shedding samples were not collected after the fourth move because some calves were given antibiotics to combat disease.

\section{Behavior}

Molasses Consumption. Molasses consumption while the calves were in isolation (STR) or individual (CTR) pens (Figure 3A) was affected by treatment $(P$ $=0.006)$ and tended to have a day $\times$ treatment interaction $(P=0.065)$. On $\mathrm{d} 2(P=0.029)$ and $\mathrm{d} 11(P=$ $0.008)$, STR calves consumed more molasses than did CTR calves. Total molasses consumed over the experiment (Figure 3B) was greater $(P=0.02)$ for STR than for CTR calves.

Increased anxiety, not seen in the cortisol concentration values, was reflected in increased molasses consumption. Increased plasma concentrations of norepinephrine increase anxiety (Shishkina et al., 2004). Norepinephrine and epinephrine stimulate glucagon release, glycogenesis, and food consumption (Ste. Marie 

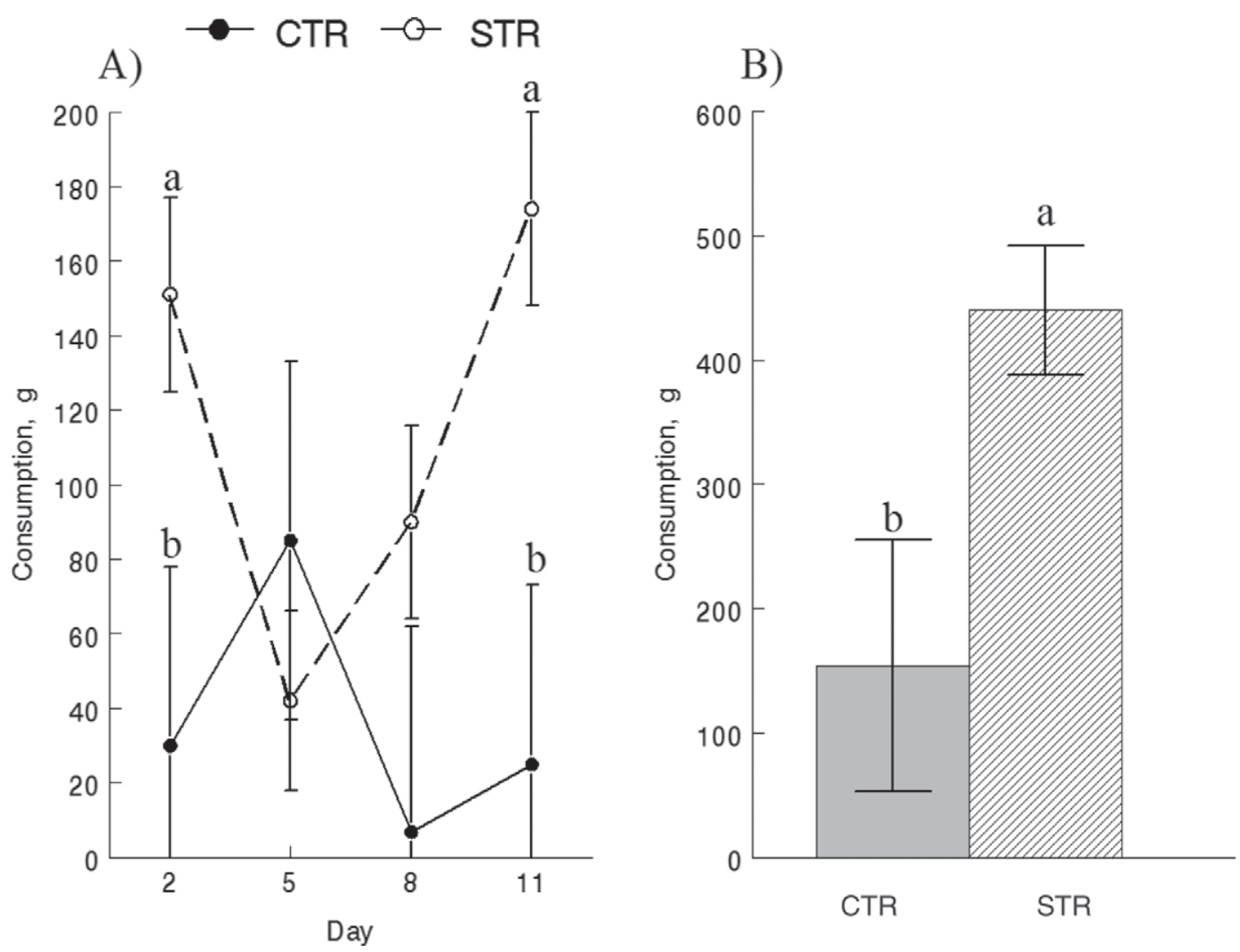

Figure 3. Mean daily molasses consumption (A) and total molasses consumption (B) of control (CTR) and social stress-treated (STR) calves. Graph means are nontransformed data. ${ }^{\mathrm{a}, \mathrm{b}}$ Means within a day with differing letters differ $P<0.05$.

and Palmiter, 2003). Psychological stress is also known to activate the reward centers and the increased desire for carbohydrate-rich comfort food (Dallman et al., 2003). Therefore, the increased glucose consumption by STR calves may be an indicator not only of HPA axis stimulation but also of sympatho-adrenal medullary axis stimulation.

Correlation coefficients of molasses consumption (during isolation) and latency to lie (in groups or control $)$ were $0.49(P=0.11)$ and $0.46(P=0.06)$ on $\mathrm{d}$ 5 and 11, respectively; however, the correlation coefficient on $\mathrm{d} 8$ was $-0.004(P=0.99)$. The correlation coefficients for molasses consumption and peak cortisol were $0.03(P=0.92),-0.08(P=0.81), 0.34(P=$ $0.19)$, and $0.61(P=0.03)$ on d $1,5,8$, and 11. Reber et al. (2006) demonstrated adrenal fatigue and associated BW and thymus weight loss in mice with a 19-d chronic intermittent psychosocial stress of social defeat and overcrowding. Accordingly, they demonstrated that cytokine secretion of inflammatory cytokines was greater in the chronic stress model. In contrast, Finger et al. (2012) found increased weight gain, intake, and caloric efficiency in mice fed a low-fat diet that were under a chronic intermittent psychosocial stress, find-

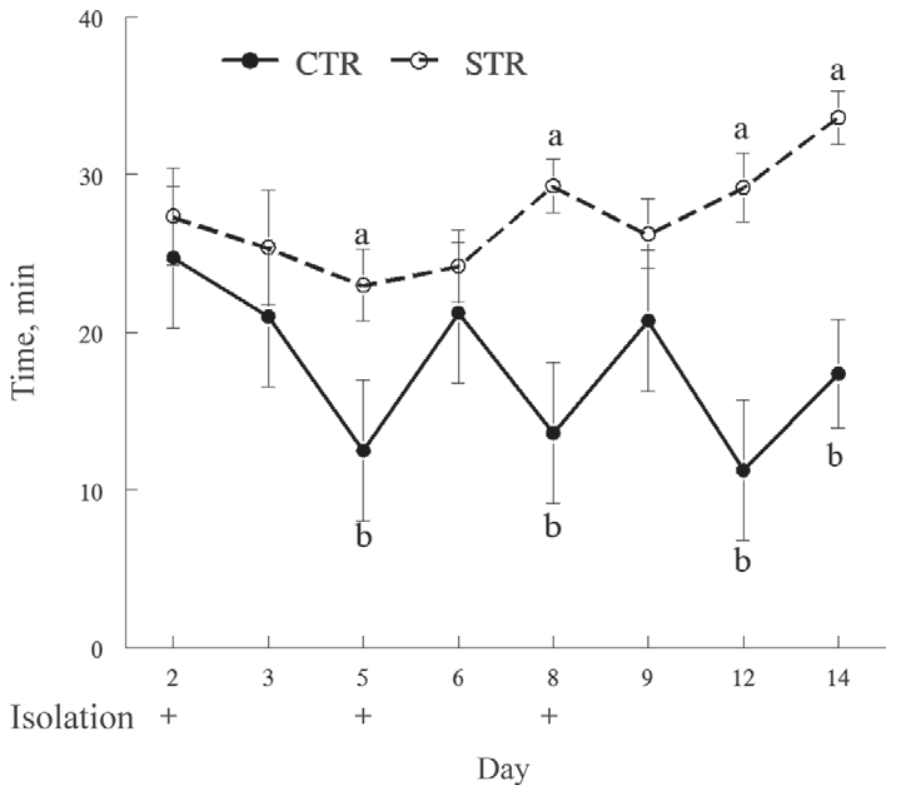

Figure 4. Latency to lie after eating for control (CTR) and social stress-treated (STR) calves. Graph means are nontransformed data. ${ }^{\mathrm{a}, \mathrm{b}}$ Means within a day with differing letters differ $(P<0.05)$. 
ings related to circulating leptin. The changes observed in the current study could be related to the increased molasses intake of the STR calves.

Latency to Lie After Eating. A treatment $(P$ $<0.001)$ effect and treatment $\times$ day interaction $(P$ $=0.04$ ) were detected for latency to lie after eating (Figure 4). Stressed calves took longer $(P=0.03)$ to lie down after eating compared with CTR calves on d $5(P=0.03)$, d $8(P<0.002), \mathrm{d} 12(P<0.003)$, and $\mathrm{d}$ $14(P<0.001)$.

Standing Behavior. Percentage of observations in which calves were standing was affected by day $(P=$ $0.015)$ and treatment $(P=0.037$; Figure $5 \mathrm{~A})$. When 4-h periods in the a.m. (Figure 5B) and p.m. (Figure $5 \mathrm{C})$ were analyzed separately, treatment $(P=0.05$ and 0.031 , for a.m. and p.m., respectively) and day $(P=$ 0.043 and 0.015 , for a.m. and p.m., respectively) effects were detected.

Standing behavior of STR calves also indicated adaptation to the stress model. Stressed calves clearly stood more than CTR calves during the first full day in group pens, after the first cycle of group to isolation and isolation to group moves. This behavior decreased to the level of the CTR calves for the next cycle but steadily increased in subsequent cycles. Our findings in the 4-h a.m. and p.m. analyses are consistent with findings in other studies in which behavioral responses of an individual animal to repeated controllable stressors depended on past experiences with that particular stressor (Bear et al., 2001), and responses were attenuated with repetition of the stressor (Vermetten and Bremner, 2002). The ACTH challenge, when analyzed over $12 \mathrm{~h}$, apparently did not produce a change in behavior of the CTR calves despite an elevated cortisol concentration. However, by comparing the 12 -h standing activity of CTR and STR calves, a disruption of circadian rhythm (principally in the afternoon) was evident. Thus, it seemed appropriate to analyze standing behavior during particular segments of the day.

During the first $4 \mathrm{~h}$ of the ACTH challenge, CTR calves stood more than on previous mornings and more than STR calves on that morning. In the $4 \mathrm{~h}$ of the afternoon on the day of ACTH challenge, CTR calves stood less than on previous afternoons, perhaps from the unaccustomed amount of activity earlier in the day. Standing behavior of STR calves indicated not only habituation to acute stress but perhaps also increased anxiety not apparent by analysis of cortisol concentrations.

It is known that the HPA system adapts easily to mild stressors; however, the sympatho-adrenal medullary system does not adapt as easily (Schommer et al., 2003). This may be why, despite an apparent adaptation
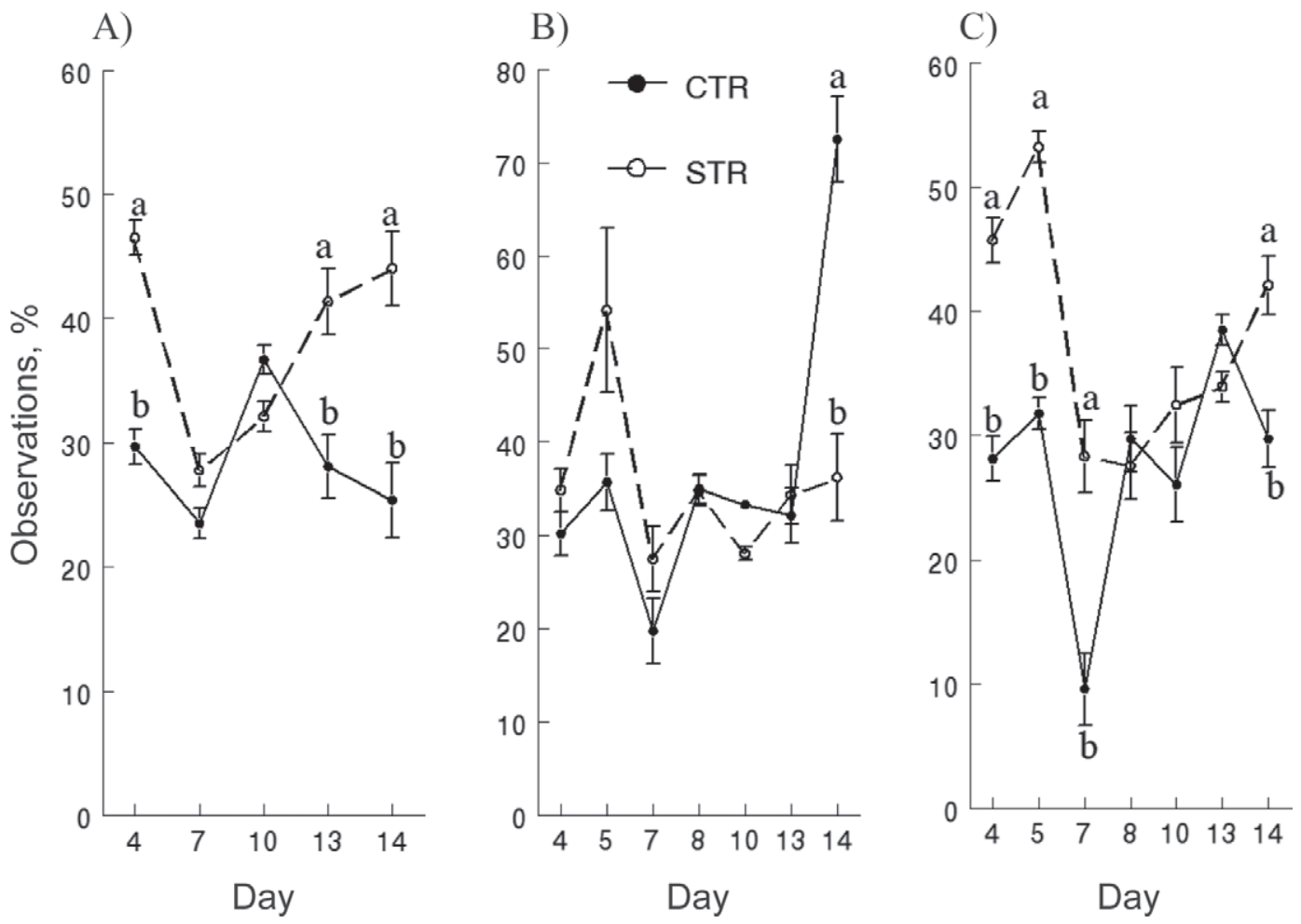

Figure 5. Mean percentage of observations of calves spent standing when calves were observed in group pens for $12 \mathrm{~h}$ (A), for $4 \mathrm{~h}$ in the morning (B), and for $4 \mathrm{~h}$ in the afternoon (C) for control (CTR) and social stress treated (STR) calves. Means are non-transformed data. ${ }_{\mathrm{a}, \mathrm{b}}$ Means within a day with differing letters differ $(P<0.05)$. 
to the stress model, mean afternoon standing behavior and latency to lie after eating in STR calves indicated increasing anxiety. Addition of a startle reaction test with a novel stimulus or measuring circulating norepinephrine or epinephrine (products of the sympathoadrenal medullary system activation) throughout the study would have helped to confirm increasing anxiety.

Molasses consumption increased in CTR calves and decreased in STR calves on d 5. This may also be due to heat stress of CTR calves and increased handling of STR calves to check and reduce body temperature. The number of Enterobacteriaceae in CTR calves showed an erratic pattern. However, analysis within STR calves showed a steady decline, indicating habituation. This pattern of wide variation is repeated in means found for behavior data. Behavior data, both the latency to lie after eating and the percentage of observations when cows were standing, gave a clear indication of habituation to acute stress but increasing anxiety with repeated applications of stress in STR animals.

This model employed 5 cycles of isolation and regrouping repeated at regular intervals. This repeated application of mild psychological stressors produced an initial acute stress reaction. This acute stress reaction diminished beginning with the second cycle of isolation and regrouping. Then, isolation and regrouping cycles 3 and 4 caused a trend of increased but muted acute stress reaction. The regular intervals at which the psychological stressors were applied seemed to habituate calves to the stressors and produced chronic stress, as intended.

\section{CONCLUSIONS}

This study was designed to determine if chronic stress could be induced by isolation and regrouping and to investigate whether molasses consumption changed as a result of chronic psychological stress. This model appeared to achieve chronic stress, as characterized by adrenal fatigue, and the stress treatment caused an increase in molasses consumption. The latter finding can be further studied and developed to be used as a noninvasive indicator of acute or chronic stressors in dairy calf research. Refinement of this stress model may be useful to study chronic stress in dairy calves. These results indicate that chronic stress may be achieved and that molasses consumption in calves may be used to verify that stress has occurred, as seen by sucrose consumption in some mouse models.

\section{REFERENCES}

Bear, M. F., B. W. Connors, and M. A. Paradiso. 2001. Neuroscience: Exploring the Brain. Lippincott, Williams and Wilkins, Baltimore, MD.
Bilandzić, N., M. Zuric, M. Lojkic, B. Simic, D. Milic, and I. Barac. 2006. Cortisol and immune measures in boars exposed to three-day administration of exogenous adrenocorticotropic hormone. Vet. Res. Commun. 30:433-444.

Brenes-Sáenz, J. C., O. R. Villagra, and J. Fornaguera Trías. 2006. Factor analysis of forced swimming test, sucrose preference test and open field test on enriched, social and isolated reared rats. Behav. Brain Res. 169:57-65.

Callaway, T. R., J. E. Keen, T. S. Edrington, L. H. Baumgard, L. Spicer, E. S. Fonda, K. Griswold, T. R. Overton, M. E. VanAmburgh, R. C. Anderson, K. J. Genovese, T. L. Poole, R. B. Harvey, and D. J. Nisbet. 2005. Fecal prevalence and diversity of Salmonella species in lactating dairy cattle in four states. J. Dairy Sci. 88:3603-3608.

Cook, S. C., and C. L. Wellman. 2004. Chronic stress alters dendritic morphology in rat medial prefrontal cortex. J. Neurobiol. 60:236-248.

Dallman, M. F., N. Pecoraro, S. F. Akana, S. E. la Fkeyr, F. Gomez, H. Houshyar, M. E. Bell, S. Bhatnagar, K. D. Laugero, and S. Manalo. 2003. Chronic stress and obesity: A new view of "comfort food". Proc. Natl. Acad. Sci. USA 100:11696-11701.

Douglas, A. J. 2005. Central noradrenergic mechanisms underlying acute stress responses of the hypothalamo-pituitary-adrenal axis: Adaptations through pregnancy and lactation. Stress 8:5-18.

Eicher, S. D., M. Schutz, F. Kearney, S. Willard, S. Bowers, S. Gandy, and K. Graves. 2007. Prepartum milking effects on parlour behaviour, endocrine and immune responses in Holstein heifers. J. Dairy Res. 74:417-424.

Fazio, E., and A. Ferlazzo. 2003. Evaluation of stress during transport. Vet. Res. Commun. 27:519-524.

Finger, B. C., T. G. Dinan, and J. F. Cryan. 2012. The temporal impact of chronic intermittent psychosocial stress on high-fat dietinduced alterations in body weight. Psychoneuroendocrinology 37:729-741.

Garnsworthy, P. C. 2005. Calf and Heifer Rearing. Nottingham University Press, Nottingham, UK.

Hickey, M. C., M. Drennan, and B. Earley. 2003. The effect of abrupt weaning of suckler calves on the plasma concentrations of cortisol, catecholamines, leukocytes, acute-phase proteins and in vitro interferon-gamma production. J. Anim. Sci. 81:2847-2855.

Kanitz, E., M. Tuchscherer, B. Puppe, A. Tuchscherer, and B. Stabenow. 2004. Consequences of repeated early isolation in domestic piglets (Sus scrofa) on their behavioural, neuroendocrine, and immunological responses. Brain Behav. Immun. 18:35-45.

Lay, D. C., Jr., H. G. Kattesh, J. E. Cunnick, M. J. Daniels, K. A. McMunn, M. J. Toscano, and M. P. Roberts. 2008. Effects of prenatal stress on sow productivity and piglet response to weaning. J. Anim. Sci. 86:1316-1324.

Madsen-Bouterse, S. A., G. J. Rosa, and J. L. Burton. 2006. Glucocorticoid modulation of Bcl-2 family members A1 and Bak during delayed spontaneous apoptosis of bovine blood neutrophils. Endocrinology 147:3826-3834.

McGuirk, S. M. 2008. Disease management of dairy calves and heifers. Vet. Clin. North Am. Food Anim. Pract. 24:139-153.

Moberg, G. P. 2000. Biological response to stress: Implications for animal welfare. Pages 1-21 in The Biology of Animal Stress. G. P. Moberg and J. A. Mench, ed. CAB International, Wallingford, UK.

Pothion, S., J. C. Bizot, F. Trovero, and C. Belzung. 2004. Strain differences in sucrose preference and in the consequences of unpredictable chronic mild stress. Behav. Brain Res. 155:135-146.

Raussi, S., A. Boissy, S. Andanson, J. Kaihilahti, and P. Prade. 2004. Is repeated regrouping stressful for heifers? Proc. 38th Int. Congr. ISAE. International Society for Applied Ethology (ISAE), Helsinki, Finland.

Reber, S. O., F. Obermeier, H. R. Straub, W. Falk, and I. D. Neumann. 2006. Chronic intermittent psychosocial stress (social defeat/overcrowding) in mice increases the severity of an acute DSS-induced colitis and impairs regeneration. Endocrinology 147:4968-4976. 
Riondato, F., A. D'Angelo, B. Miniscalco, C. Bellino, and R. Guglielmino. 2008. Effects of road transportation on lymphocyte subset in calves. Vet. J. 175:364-368.

Sapolsky, R. M. 2004. Homeostasis plus: The more stress appropriate concept of allostasis. Pages 8-9 in Why Zebras Don't Get Ulcers. R. M. Sapolsky, ed. Henry Holt and Co. Ltd., New York, NY.

Schommer, N. C., D. H. Hellhammer, and C. Kirschbaum. 2003. Dissociation between reactivity of the hypothalamus-pituitary-adrenal axis and the sympathetic-adrenal-medullary system to repeated psychosocial stress. Psychosom. Med. 65:450-460.

Shishkina, G. T., T. S. Kalinina, and N. Dygalo. 2004. Attenuation of $\alpha_{2 \mathrm{~A}}$-adrenergic receptor expression in neonatal rat brain by RNA interference or antisense oligonucleotide reduced anxiety in adulthood. Neuroscience 129:521-528.

Ste. Marie, L., and R. D. Palmiter. 2003. Norepinephrine and epinephrine-deficient mice are hyperinsulinemic and have lower blood glucose. Endocrinology 144:4427-4432.

USDA-APHIS (US Department of Agriculture Animal and Plant Health Inspection Service). 2008. Colostrum feeding and management on U.S. dairy operations. Centers for Epidemiology and Animal Health, USDA-APHIS-VS, Fort Collins, CO.
Veissier, I., A. Boissy, A. M. dePassillé, J. Rushen, C. G. van Reenen, S. Roussel, S. Andanson, and P. Pradel. 2001. Calves' responses to repeated social regrouping and relocation. J. Anim. Sci. 79:25802593.

Vermetten, E., and J. D. Bremner. 2002. Circuits and systems in stress. I: Preclinical studies. Depress. Anxiety 15:126-147.

Wattiaux, M. A. 2000. Heifer raising-birth to weaning; Overview of sound management practices. Chapter 25 in Dairy Essentials. Babcock Institute, University of Wisconsin, Madison.

Wilcox, C. S., M. M. Schutz, S. S. Donkin, D. C. Lay Jr., and S. D. Eicher. 2008. Effect of temporary glycosuria on molasses consumption in Holstein calves. J. Dairy Sci. 91:3607-3610.

Williams, J. L., J. E. Minton, J. A. Patterson, J. Marchant Forde, and S. D. Eicher. 2008. Lairage during transport of eighteen-kilogram pigs has an impact on innate immunity and commensal bacteria diversity in the intestines. J. Anim. Sci. 86:1232-1244.

Yeh, S.-T. 2002. Using trapezoidal rule for the area under a curve calculation. Page 229 in Proc. SAS User's Group International (SUGI 27), Orlando, FL. SAS Institute Inc., Cary, NC. 